Мнение врачей о необходимости и сроках отказа от курения перед плановым кардиохирургическим вмешательством

\author{
Е.Д. Баздырев ${ }^{\varpi}$, Н.А. Галимова, О.Л. Барбараш \\ ФГБНУ «Научно-исследовательский институт комплексных проблем сердечно-сосудистых заболеваний», Кемерово, Россия
}

\begin{abstract}
Аннотация
Обоснование. В настоящее время нет официальных рекомендаций по срокам отказа от курения пациентов перед кардиохирургическим вмешательством. И хотя, по данным большинства экспертных российских и зарубежных документов, продолжение курения является прогностически неблагоприятным фактором, в руководствах не указаны сроки отказа от курения, позволяющие минимизировать послеоперационные осложнения и улучшить прогноз данной категории пациентов.

Цель. Анализ мнений врачей различных специальностей о необходимости и сроках отказа от курения пациентов перед плановым вмешательством на сердце и сосудах.

Материал и методы. Проведено анкетирование 320 специалистов терапевтического - 250 (78,1\%) - и хирургического - 70 (21,9\%) - профилей со стажем работы не менее 10 лет, выполняющих предоперационную подготовку пациентов к коронарному шунтированию, вмешательство и послеоперационную реабилитацию. Анкета включала 7 вопросов, позволяющих отразить мнение врача о целесообразности и времени, а также лимитирующих факторах отказа от курения у пациентов, направленных для планового выполнения коронарного шунтирования.

Результаты. Большая часть респондентов, 218 (68,1\%) человек, рекомендовали бы пациентам отказаться от курения в период, предшествующий операции; вдвое меньше специалистов, 102 (31,9\%) врача, посоветовали бы отказаться от курения после хирургического вмешательства. Большинство опрошенных, 166 (76,2\%) врачей, считают, что пациентам следует отказаться от употребления табачной продукции за 2 мес до планируемой операции. 1/3 респондентов отметили целесообразным отказ после оперативного лечения: 31 (30,4\%) специалист рекомендовал это сделать сразу после лечения, 43 (42,1\%) - в течение 1-го месяца. Причиной, почему специалисты не рекомендовали бы пациенту отказ от курения в период, близкий к хирургическому вмешательству, большинство анкетируемых назвали повышенный риск ухудшения клинического статуса больного: усиление кашля - 200 (62,5\%) специалистов, секреции мокроты - 85 (26,6\%), одышки - 25 (7,8\%); $10(3,1 \%)$ врачей отметили риск развития абстинентного синдрома и стресса.

Заключение. У врачей, занимающихся подготовкой и ведением кардиохирургических пациентов, отсутствует единое мнение о сроках и периоде отказа от употребления табачной продукции.
\end{abstract}

Ключевые слова: ишемическая болезнь сердца, курение, отказ от курения, кардиохирургическое вмешательство

Для цитирования: Баздырев Е.Д., Галимова Н.А., Барбараш 0.Л. Мнение врачей о необходимости и сроках отказа от курения перед плановым кардиохирургическим вмешательством. СardioСоматика. 2021; 12 (1): 34-40. DOI: 10.26442/22217185.2021.1.200757

\section{Введение}

В настоящее время доказано, что курение является одним из наиболее распространенных факторов риска развития и прогрессирования сердечно-сосудистых заболеваний. По данным опроca GATS 2016 г., в России 30,5\% (36,4 млн) взрослого населения постоянно употребляют табак в любом виде, из них курительный табак - 30,3\% (36,3 млн): 49,5\% мужчин и 14,4\% женщин [1].

Крайне актуальна проблема курения с точки зрения влияния на ближайшие и отдаленные результаты хирургического лечения пациентов с атеросклерозом любого сосудистого бассейна. Пациенты, сохраняющие эту вредную привычку в послеоперационном периоде, имеют более высокий риск развития повторных ишемических событий в реваскуляризированных и ранее не реваскуляризированных бассейнах, более высокий риск нарушений ритма сердца (фибрилляции предсердий) и проявлений

\section{Информация об авторах / Information about the authors}

Баздырев Евгений Дмитриевич - д-р мед. наук, зав. лаб. эпидемиологии сердечно-сосудистых заболеваний НИИ КПСС3. E-mail: edb624@mail.ru: ORCID: 0000-0002-3023-6239

Галимова Наталья Александровна - аспирант НИИ КПССЗ. ORCID: 0000-0003-4948-2429

Барбараш Ольга Леонидовна - чл.-Кор. РАН, д-р мед. наук, проф., дир. НИИ КПСС3. ORCID: 0000-0002-4642-3610 сердечной недостаточности [2-5]. Логичным выглядит утверждение о том, что пациенты с любыми проявлениями атеросклероза, в том числе требующими хирургической коррекции, должны быть информированы о необходимости прекращения курения.

В настоящее время нет четких рекомендаций по срокам отказа от курения перед кардиохирургическим вмешательством. Большинство официальных документов декларирует, что продолжение курения является прогностически неблагоприятным фактором [6, 7]. Однако, к сожалению, в экспертных документах не приводятся сроки отказа от курения, позволяющие минимизировать послеоперационные осложнения и улучшить прогноз данной категории пациентов. Вероятнее всего, с одной стороны, это связано с небольшим количеством исследований, посвященных данной проблеме, а также противоречивостью опубликованных

Evgeny D. Bazdyrev - D. Sci. (Med.), Research Institute for Complex Issues of Cardiovascular Diseases. E-mail: edb624@mail.ru; ORCID: 0000-0002-3023-6239

Natalia A. Galimova - Graduate Student, Research Institute for Complex Issues of Cardiovascular Diseases. ORCID: 0000-0003-4948-2429

Olga L. Barbarash - D. Sci. (Med.), Prof., Corr. Memb. RAS, Research Institute for Complex Issues of Cardiovascular Diseases.

ORCID: 0000-0002-4642-3610 


\title{
The necessity and timing of smoking cessation before scheduled cardiovascular interventions - doctors' survey
}

\author{
Evgeny D. Bazdyrev ${ }^{\circledR}$, Natalia A. Galimova, Olga L. Barbarash
}

Research Institute for Complex Issues of Cardiovascular Diseases, Kemerovo, Russia

\begin{abstract}
Background. No specific time frames are specified in the guidelines for smoking cessation for patients preparing for cardiovascular intervention. Current smoking is an adverse prognostic factor according to the Russian and foreignlegal documents, but the recommendations do not specify the timing of smoking cessation, even though it could help minimize postoperative complications and improve the prognosis.

Aim. To analyze doctors' opinions on the necessity and timing of smoking cessation for patients before scheduled cardiovascular surgery. Material and methods. 320 specialists - 250 (78.1\%) general practitioners and $70(21.9 \%)$ surgeons, were surveyed, each participant had at least 10 years of experience performing preoperative program before coronary artery bypass graft surgeries or other interventions and providing postoperative rehabilitation. The questionnaire included seven questions reflecting the doctors' opinion on the feasibility, timing andlimiting factors of smoking cessation in patients referred for planned coronary artery bypass graft surgery.

Results. Most of the respondents - $218(68.1 \%)$ people would recommend patients to stop smoking during preoperative period; fewer specialists $102(31.9 \%)$, would advise them to quit smoking after surgery. The majority of respondents, $166(76.2 \%)$ doctors, believe that patients should quit smoking 2 months before the operation. A third of respondents said it would be appropriate to stop smoking after surgical treatment: 31 (30.4\%) specialists recommended it immediately after the operation; $43(42.1 \%)$ - within the first month. The majority of respondents noted an increased risk of patient's health deterioration, such as: increased cough - according to 200 (62.5\%) specialists; sputum secretion - 85 (26.6\%); shortness of breath $-25(7.8 \%)$; and $10(3.1 \%)$ doctors mentioned the risk of developing withdrawal syndrome and anxiety. All of those are the reasons why experts consider not recommending patient to stop smoking during preoperative period.

Conclusion. Doctors, involved in the caring for and management of cardiac surgery patients, do not have unanimous opinion on the time frames of smoking cessation.
\end{abstract}

Keywords: coronary artery disease, smoking, smoking cessation, cardiovascular intervention

For citation: Bazdyrev ED, Galimova NA, Barbarash OL. The necessity and timing of smoking cessation before scheduled cardiovascular interventions - doctors' survey. Cardiosomatics. 2021; 12 (1): 34-40. DOI: 10.26442/22217185.2021.1.200757

результатов [8-13]. С другой стороны, у практикующих врачей нет убежденности в необходимости отказа от курения в периоперационном периоде. Считается, что отказ от курения накануне операции может спровоцировать обострение хронических заболеваний легких - ухудшение респираторной симптоматики в виде усиления кашля и секреции мокроты, что негативно отразится на течении послеоперационного периода. Обсуждается и то, что предоперационный отказ от курения может привести к несостоятельности швов грудины, нестабильности и расхождению ее фрагментов. Однако данные ранее опубликованных работ показали, что все эти риски в большей степени характерны для курящих на момент лечения пациентов [8-11, 14].

Цель исследования - анализ мнения врачей различных специальностей о необходимости и сроках отказа от курения пациентов перед плановым вмешательством на сердце и сосудах.

\section{Материал и методы}

Во время VIII Съезда кардиологов Сибирского федерального округа 2019 г. в Кемерове проведено анкетирование 320 врачей, принимающих непосредственное участие в подготовке и проведении коронарного шунтирования либо в послеоперационном ведении пациентов. Другое условие участия в опросе - стаж работы (на момент анкетирования) 10 и более лет. Вопросы анкеты позволяли проанализировать мнение врачей о необходимости и рекомендуемых ими сроках отказа от курения пациентов перед плановым кардиохирургическим вмешательством, а также выявить ограничивающие факторы (см. таблицу).

Статистическую обработку результатов проводили с использованием пакета прикладных программ Statistica 6.0. Анализ показателей, измеренных в качественных шкалах, представлен в виде распределения частот $(n, \%)$. Возраст и стаж работы респондентов представлены в виде среднего значения и стандартного отклонения.

\section{Результаты}

В анкетировании приняли участие 250 (78,1\%) врачей терапевтического профиля - 200 (62,5\%) кардиологов, 50 (15,6\%) терапевтов и 70 (21,9\%) врачей хирургического профиля: 54 (16,9\%) сердечно-сосудистых хирурга, 16 (5\%) анестезиологов-реаниматологов. Среди принявших участие в опросе большая часть врачи женского пола: 204 (63,8\%) против 116 (36,2\%); средний возраст респондентов составил 40,2 23,4 года, стаж работы по специальности - 17,1 2,8 года.

Первый вопрос анкеты направлен на выяснение мнения врачей о необходимости отказа пациентов от курения перед запланированной кардиохирургической операцией. Большая часть специалистов, 218 (68,1\%) человек, рекомендовали бы пациенту отказаться от курения перед операцией. В 2 раза меньше врачей, 102 (31,9\%) специалиста, посоветовали бы пациентам отказаться от данной привычки уже после вмешательства.

Из 250 представителей терапевтической специальности (терапевты, кардиологи) 178 (71,2\%) рекомендовали бы отказаться от курения до оперативного вмешательства, 72 (28,8\%) специалиста после вмешательства. Из 70 врачей хирургической специальности (кардиохирурги, анестезиологи-реаниматологи), принявших участие в анкетировании, 40 (57,1\%) рекомендовали бы бросить курение до коронарного шунтирования, 30 (42,9\%) - после.

Большая часть опрошенных рекомендовали отказаться от курения за 2 мес до планируемого вмешательства - 166 (76,2\%) врачей, за 1 мес - $26(11,9 \%)$, за 3 нед - 4 (1,8\%), за 2 нед - 4 (1,8\%) и накануне операции - 18 (8,3\%) специалистов.

Из 102 врачей, считающих целесообразным отказ от употребления табачной продукции после операции, большинство посоветовали бы отказаться в первые дни после вмешательства - 31 (30,4\%) специалист, через 2 нед - 20 (19,6\%), через 1 мес - 23 (22,5\%), че- 


\begin{tabular}{|c|c|c|}
\hline Анке & Іля анализа мнения врачей о необходимости отказа от курения пацие & тов, готовящихся к вмешательству на сердце или сосудах \\
\hline № & Вопрос & Варианты ответа \\
\hline 1 & Укажите свою специальность & \\
\hline 2 & $\begin{array}{l}\text { Курящему пациенту планируется кардиохирургическое вме- } \\
\text { шательство. Когда, по вашему мнению, ему следует отказаться }\end{array}$ & $\begin{array}{l}\text { А. Перед операцией (если вы отметили данный пункт, переходите } \\
\text { к вопросу №3) }\end{array}$ \\
\hline & o курения? & $\begin{array}{l}\text { Б. После хирургического вмешательства (если вы выбрали дан- } \\
\text { ный пункт, переходите к вопросу №4) }\end{array}$ \\
\hline 3 & В какие сроки перед планируемым кардиохирургическим вме- & A. За 2 мес \\
\hline & $\begin{array}{l}\text { шательством вы оы порекомендовали пациенту отказаться } \\
\text { от курения? }\end{array}$ & Б. За 1 мес \\
\hline & & В. За 3 нед \\
\hline & & Г. За 2 нед \\
\hline & & Д. Накануне оперативного вмешательства \\
\hline 4 & В какие сроки после кардиохирургического вмешательства & А. Сразу после вмешательства \\
\hline & вы оы порекомендовали пациенту отказаться ол курения? & Б. Через 2 нед \\
\hline & & В. Через 1 мес \\
\hline & & Г. Через 2 мес \\
\hline & & Д. Через 3 мес \\
\hline 5 & Несмотря на ранее выбранный ответ, вы бы рекомендова- & А. Да \\
\hline & $\begin{array}{l}\text { ли пациенту отказаться от курения во время госпитализации } \\
\text { для кардиохирургического вмешательства? }\end{array}$ & Б. Нет \\
\hline 6 & Несмотря на ранее выбранный ответ, вы бы рекомендовали паци- & А. Да \\
\hline & $\begin{array}{l}\text { енту отказаться от курения сразу (в первые дни) после кардиохи- } \\
\text { рургического вмешательства? }\end{array}$ & Б. Нет \\
\hline 7 & Несмотря на ранее выбранный ответ, по какой причине вы бы & А. Из-за опасения усиления кашля \\
\hline & $\begin{array}{l}\text { не стали рекомендовать пациенту отказаться от курения накануне } \\
\text { кардиохирургического вмешательства? }\end{array}$ & Б. Из-за опасения усиления секреции мокроты \\
\hline & & В. Из-за опасения усиления одышки \\
\hline & & Г. Другое (укажите причину) \\
\hline
\end{tabular}

рез 2 мес - 18 (17,7\%) и 3 мес - 10 (9,8\%) врачей. Причем среди анкетируемых, рекомендующих отказаться от курения через 3 мес, врачи исключительно хирургической специальности.

Мнения врачей о возможности отказа от курения в момент поступления на операцию разделились: 53,1\% специалистов ответили отрицательно, 46,9\% - положительно. Интересно, что врачи терапевтического профиля в равной степени проголосовали за отказ от курения и против него, в то время как большинство врачей хирургической специальности не рекомендовали бы этого делать (за - 24 человека, против - 46). Более подробно распределение ответов на данный вопрос представлено на рис. 1.

Аналогичное отношение анкетируемых к отказу от курения сразу после оперативного лечения: 184 (57,5\%) респондента из 320 участвовавших в опросе ответили отрицательно (рис. 2).

Причиной, почему не рекомендовали бы отказ от курения в период, близкий к хирургическому вмешательству, большинство врачей указали повышенный риск ухудшения клинического статуса пациентов: усиление кашля - 200 (62,5\%) опрошенных, секреции мокроты - 85 (26,6\%), одышки - 25 (7,8\%); 10 (3,1\%) врачей отметили риск развития абстинентного синдрома и стресса (рис. 3).

Таким образом, большинство респондентов рекомендовали бы отказаться от курения не позднее 2 мес до планируемого кардиохирургического вмешательства. 1/3 опрошенных выбрали вариант отказа от курения после операции, причем большая часть врачей (30,4\%) - сразу после операции; несколько меньше, 22,5\% специалистов, - через 1 мес после вмешательства. Больше $1 / 2$ респондентов не рекомендовали бы отказ от употребления табачной продукции в первые дни после операции по причине опасения ухудшения респираторной симптоматики - усиления кашля и секреции мокроты.

\section{Обсуждение}

В различных исследованиях [8-11, 14] доказано, что курение является фактором развития как периоперационных, так и ближайших и отдаленных послеоперационных осложнений при многих видах хирургической помощи. У пациентов общей 


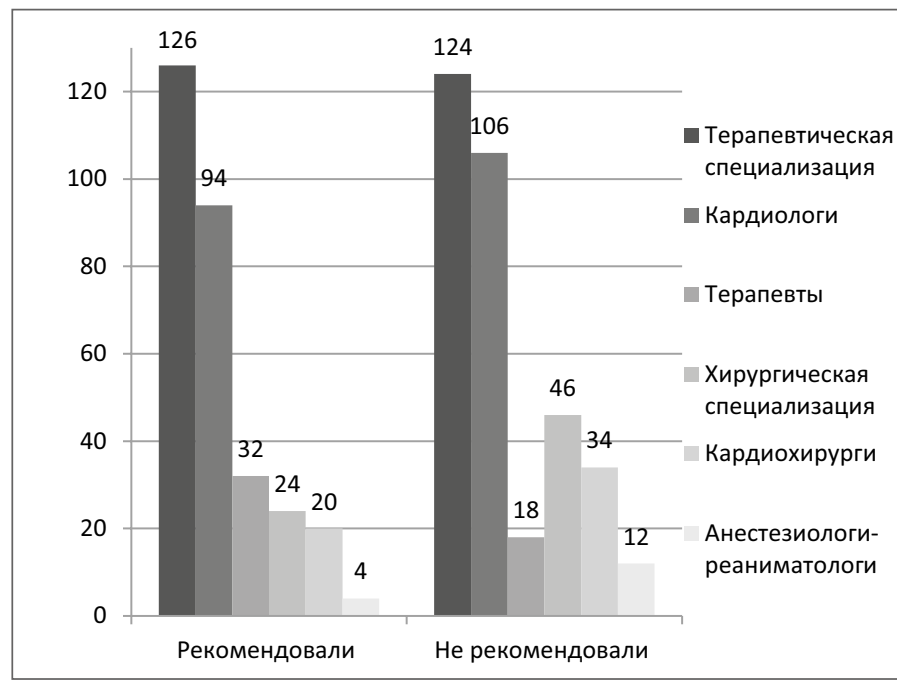

Рис. 1. Распределение ответов респондентов об отказе от курения накануне или во время госпитализации в зависимости от врачебной специализации (n).

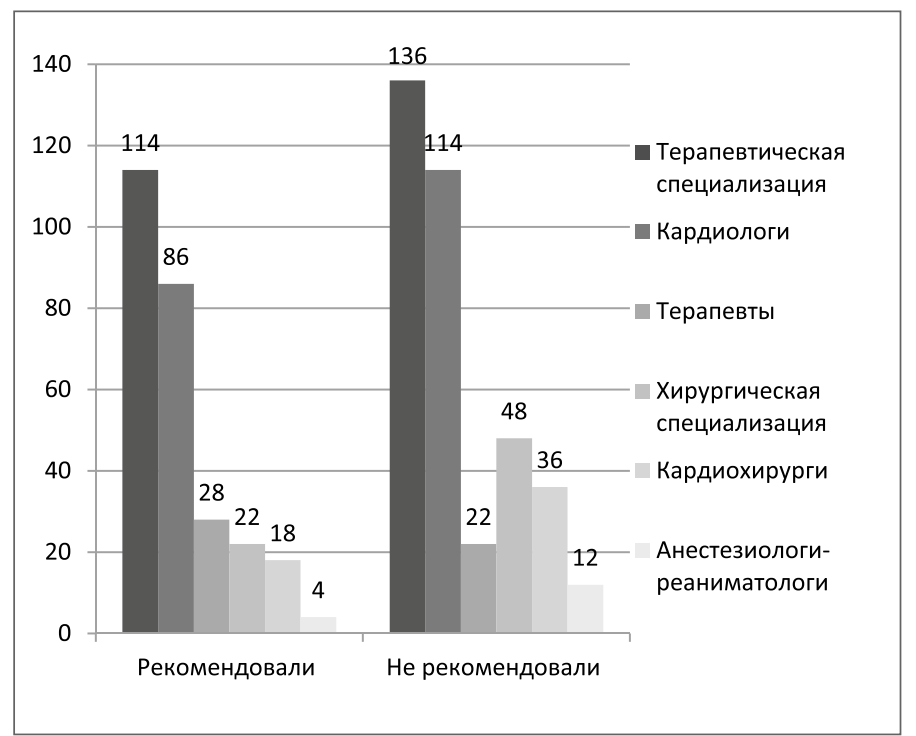

Рис. 2. Распределение ответов респондентов об отказе от курения в первые дни после кардиохирургического вмешательства в зависимости от врачебной специализации (n).

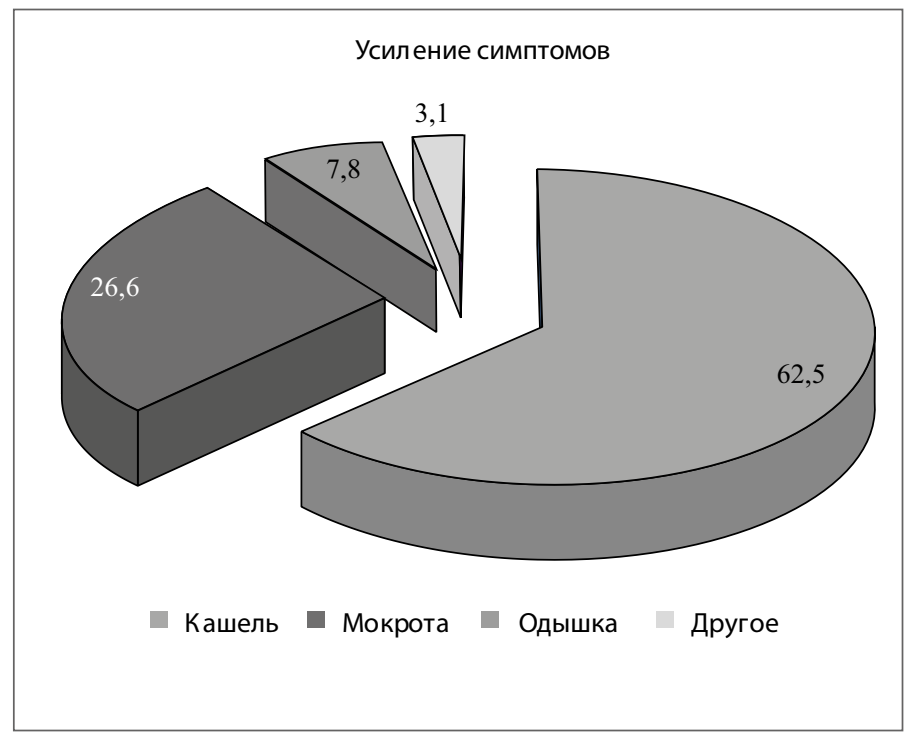

Рис. 3. Опасения врачей, связанные с отказом пациентов от курения накануне кардиохирургического вмешательства (\%). хирургии курение ассоциировано с увеличением риска развития поверхностной и глубокой инфекции области хирургического вмешательства, сепсиса, инфаркта миокарда, инсульта, пневмонии, продленной искусственной вентиляции легких. При вмешательствах на сердце и сосудах курение обусловливает повышение риска развития бронхолегочных осложнений, стернальной инфекции, дисфункции венозного шунта, более длительной искусственной вентиляции легких. При оперативных методах лечения в ортопедии курение увеличивает риск не только инсульта, пневмонии, инфекции области хирургического вмешательства, но ассоциируется с нарушением процесса репарации костей, увеличением продолжительности послеоперационного периода и более выраженным болевым синдромом. Негативное влияние курения отмечено и в пластической хирургии: увеличение послеоперационных рубцов, более медленное заживление ран, а также дисфункция имплантата при реконструкции груди.

По данным многих исследований, у курящих пациентов выше риск периоперационной смертности в сравнении с некурящими: отношение шансов (ОШ) 1,17, 95\% доверительный интервал (ДИ) 1,10-1,24. Курящие пациенты имеют более высокую вероятность кардиальных осложнений (0Ш 1,77, 95\% ДИ 1,57-1,99), а также острых респираторных расстройств (0Ш 1,73, 95\% ДИ 1,35-2,23). Статус «активно курящие» ассоциирован с более длительным пребыванием в отделении реанимации (0Ш 1,60, 95\% ДИ 1,14-2,25) и развитием пневмонии (0Ш 1,50, 95\% ДИ 1,43-1,59). Кроме этого, риск стернальной дегисценции (несостоятельность швов грудины, прорезывание швов, нестабильность грудины, расхождение ее фрагментов), задержка консолидации грудины, инфекции области хирургического вмешательства у активно курящих пациентов также выше в сравнении с некурящими (0Ш 2,15, 95\% ДИ 1,87-2,49; ОШ 2,15, 95\% ДИ 1,58-2,09 и ОШ 1,29, 95\% ДИ 1,18-1,40 соответственно) [8-11].

При этом исследования, посвященные проблеме отказа от курения перед хирургическим лечением, демонстрируют противоречивые результаты. В работах Møller и соавт. 2002 г. (отказ за 8 нед до хирургического вмешательства), Lindström и др. 2008 г. (отказ за 4 нед до вмешательства) и Shi и соавт. 2013 г. (отказ за 4 нед до вмешательства) показан высокий процент осложнений послеоперационного периода у пациентов, продолжавших курить. В исследованиях Thomsen и соавт. 2003 г. (отказ за 3 нед до хирургического вмешательства), Sørensen и соавт. 2007 г. (отказ за 4 нед до вмешательства), Thomsen и соавт. 2010 г. (отказ за 4 нед до вмешательства) послеоперационные результаты лечения курящих пациентов в сравнении с некурящими не имели статистически значимой разницы [12].

Результаты рандомизированного исследования 168 пациентов по оценке влияния программы периоперационного отказа от курения на показатели прекращения/сокращения курения и периоперационные осложнения продемонстрировали отсутствие статистически значимых различий в развитии интра- и послеоперационных (как в раннем, так и через 30 дней после оперативного лечения) осложнений среди пациентов, которые получали никотинзамещающую терапию (т.е. не курили) в течение 6 нед, в сравнении с курящими [13].

Таким образом, анализ литературы, посвященный вопросам сроков отказа от курения перед хирургическим вмешательством, не дает однозначного ответа. В ряде исследований продемонстрировано, что отказ от курения в течение нескольких недель до операции либо не имеет преимуществ перед его продолжением [13], либо увеличивает риск осложнений [12]. Существует и противоположная точка зрения [8-11]. Однако важно понимать, что некоторые значимые неблагоприятные эффекты, вызванные никотином и угарным газом, могут быть прекращены в течение 24-48 ч после прекращения курения $[15,16]$. 
Наблюдательные исследования показывают, что наиболее благоприятным является воздержание от употребления табачной продукции в течение не менее 2 мес до операции, что согласуется с результатами представленного в нашем исследовании опроса [17]. Так, 68,1\% кузбасских врачей рекомендовали бы пациентам отказаться от курения до оперативного лечения, причем 76,2\% опрошенных считают, что сроки отказа от курения должны быть не менее чем за 8 нед (2 мес) до планируемой операции.

Следующий вывод проведенного опроса заключается в том, что большинство врачей не рекомендовали бы пациентам отказываться от курения в период госпитализации (53,1 против $46,9 \%)$ из-за опасения усиления кашля $(62,5 \%)$ и продукции мокроты $(26,6 \%)$. Действительно, усиление продукции мокроты и, соответственно, кашля может негативно сказываться на процессах заживления раны после стернотомии. Но ухудшение данной симптоматики отчасти является закономерным процессом (за счет длительного нахождения в горизонтальном положении, поверхностного дыхания из-за болевого синдрома и др.) у лиц, имеющих бронхолегочную патологию, в том числе развивающуюся при длительном курении. В соответствии с клиническими рекомендациями Минздрава России «Синдром зависимости от табака, синдром отмены табака у взрослых» [18], при выявлении респираторных симптомов (кашель, мокрота) рекомендовано назначение муколитических или отхаркивающих препаратов, что, вероятно, поможет нивелировать данную респираторную симптоматику.

Из-за отсутствия хорошо спланированных и выполненных на высоком доказательном уровне рандомизированных исследований вопрос сроков отказа от курения перед плановым хирургическим вмешательством не отражен в экспертных отечественных и зарубежных документах.

Согласно российским клиническим рекомендациям 2016 г. «Коронарное шунтирование больных ишемической болезнью сердца: реабилитация и вторичная профилактика» [6], во время госпитализации все курильщики должны быть включены в программу по отказу от курения (уровень доказательности 1A), а также получать неоднократные советы по отказу от курения и применению никотинзамещающей терапии (уровень доказательности 1А). При этом данные о сроках отказа от курения при вмешательствах на сердце и сосудах отсутствуют.

В клинических рекомендациях Федерации анестезиологов-реаниматологов России 2018 г. «Периоперационное ведение пациентов с дыхательной недостаточностью» [15] представлены плюсы и минусы отказа от курения в разные сроки перед хирургическим вмешательством у пациентов с проявлением дыхательной недостаточности. Авторы утверждают, что в срок отказа от курения за 2-3 дня до оперативного лечения нормализуются уровни угарного газа (карбоксигемоглобина) и никотина, снижается частота интраоперационной ишемии миокарда и улучшается дренажная функция бронхов. При этом опасения специалистов связаны с повышением реактивности дыхательных путей, что может обусловливать риск интра- и послеоперационного бронхоспазма, ларингоспазма. Кроме этого, отказ от курения (за 2-3 дня до операции) может спровоцировать усугубление острых респираторных заболеваний, увеличение продукции мокроты, а также повышение риска тромбоза глубоких вен, раздражительность и беспокойство.

В рекомендациях Канадского общества сердечно-сосудистых хирургов 2017 г. по периоперационной оценке сердечно-сосудистого риска и ведению пациентов, перенесших некардиальную операцию, несмотря на высокий уровень экспертных заключений, но низкую доказательность (IC), приводятся следующие положения: поскольку даже краткие консультации по отказу от курения во время предоперационной оценки могут положительно влиять на прекращение употребления табака, авторы считают, что важно использовать эту возможность для оптимизации долгосрочного сердечного риска [19].

В рекомендациях Общества по усиленному восстановлению после операции 2015 г. по предоперационной и интраоперационной помощи в гинекологической/онкологической хирургии авторы также отмечают, что курение связано с высоким риском послеоперационных осложнений. Результат отказа от курения будет более благоприятным, если пациент воздержится от никотина в течение 4 нед до оперативного вмешательства. При этом в руководстве указано, что поведенческая поддержка и заместительная никотиновая терапия приводят к кратковременному периоду прекращению курения и имеют слабую доказательную базу, но, несмотря на это, могут снижать послеоперационную заболеваемость [20].

В рекомендациях Американского общества сосудистой хирургии по уходу за пациентами с аневризмой брюшной аорты (2018 г.), несмотря на экспертность, но низкую доказательность (IC), приводятся данные о необходимости отказа от курения как минимум за 2 нед до реконструктивного лечения аневризмы брюшного отдела аорты [21].

В 2017 г., основываясь на результатах метаанализов, систематических обзоров и крупных когортных исследований, Французское общество анестезии и интенсивной терапии опубликовало руководство по контролю курения в периоперационном периоде [22]. Французское общество анестезии и интенсивной терапии рекомендует (IA) отказаться от курения независимо от сроков вмешательства; при этом чем продолжительнее период воздержания от курения, тем выше польза. Кроме этого, авторы рекомендуют предлагать поведенческое лечение и назначать никотинзамещающую терапию для прекращения курения перед любым запланированным хирургическим вмешательством. Также в руководстве сделан акцент на том, что все специалисты, участвующие в оказании медицинской помощи (хирурги, анестезиологи-реаниматологи и др.), должны информировать курящих пациентов о положительных эффектах отказа от курения и предлагать им специальное руководство и индивидуальное наблюдение [22].

В настоящее время убедительно доказано, что пациенты с большей серьезностью относятся к прекращению курения в связи с определенными событиями. В качестве триггера отказа от курения могут выступать беременность, диагностика заболеваний, госпитализация, а также планируемое хирургическое вмешательство. В ряде исследований [17] продемонстрировано увеличение частоты самопроизвольного прекращения курения у данной категории лиц по сравнению с показателями среди населения в целом. Среди госпитализированных пациентов шансы бросить курить увеличиваются с интенсивностью медицинских вмешательств по поводу основного заболевания.

Планируемое на сердце или сосудах вмешательство является поводом для пациента отказаться от курения для снижения периоперационных осложнений и улучшения отдаленного послеоперационного прогноза.

Несмотря на отсутствие официальных рекомендаций о сроках отказа от курения перед запланированным хирургическим вмешательством, результаты исследований и данные экспертных документов при невысоком уровне доказательности свидетельствуют о необходимости как можно более раннего отказа пациентов от курения с целью снижения рисков неблагоприятного прогноза.

В связи с этим участвующие в ведении пациентов врачи должны определять статус курения пациента, информировать о рисках курения и предлагать различные методы воздействия, а при необходимости - назначать никотинзамещающую терапию для более раннего отказа пациента до предполагаемых сроков хирургиче- 
ского вмешательства. Все клиницисты должны занять активную позицию в отношении рекомендаций по отказу от курения среди пациентов. Ключевой шаг в усилиях по прекращению курения заключается не только в рекомендациях терапевтов (кардиологов), но анестезиологов и хирургов.

Настоящее исследование имеет ряд ограничений. Во-первых, не приведен расчет необходимого числа респондентов для дальнейшего статистического анализа, так как предполагался лишь опрос. Во-вторых, анализируемые группы врачей терапевтической и хирургической специальностей не сопоставимы по численности. В третьих, не учтен статус курения самих медицинских работников.

\section{Заключение}

Результаты опроса показали, что отказ от курения - необходимая мера предотвращения неблагоприятного исхода у пациентов, готовящихся к плановой операции на сердце и сосудах.
Однако у врачей, занимающихся подготовкой и ведением пациентов сердечно-сосудистого профиля, отсутствует единое мнение о сроках и периоде отказа от употребления табачной продукции, что требует дальнейшего исследования.

Конфликт интересов. Авторы заявляют об отсутствии конфликта интересов.

Conflict of interests. The authors declare no conflict of interest.

Вклад авторов. Все авторы подтверждают соответствие своего авторства, согласно международным критериям ICMJE.

Authors' contribution. All authors confirm the compliance of their authorship according to the international ICMJE criteria.

Источник финансирования. Авторы заявляют об отсутствии внешнего финансирования при проведении исследования.

Funding source. This study was not supported by any external sources of funding.

\section{СПИСОК СОКРАЩЕНИЙ}

ДИ - доверительный интервал

ОШ - отношение шансов

\section{Литература/References}

1. Глобальный опрос взрослого населения о потреблении табака: Российская Федерация. Краткий обзор. 2016. Режим доступа: http://www.euro.who.int/ru/health-topics/disease-prevention/ tobacco/publications/2017/global-adult-tobacco-survey-russianfederation.-executive-summary-2016-2017. Ссылка активна на 10.06.2020 [Global Adult Tobacco Survey: Russian Federation. Executive Summary. 2016. Available at: http://www.euro.who.int/ ru/health-topics/disease-prevention/tobacco/publications/2017/ global-adult-tobacco-survey-russian-federation.-executivesummary-2016-2017. Accessed: 10.06.2020 (in Russian)].

2. Самородская И.В., Баздырев Е.Д., Барбараш О.Л. «Парадокс» факторов риска развития сердечно-сосудистых заболеваний. Фокус на курение. Комплексные проблемы сердечно-сосудистых заболеваний. 2019; 8 (1): 90-9 [Samorodskaya I.V., Bazdyrev E.D., Barbarash O.L. Cardiovascular risk factor paradox. A focus on smoking. Complex Issues of Cardiovascular Diseases. 2019; 8 (1): 90-9 (in Russian)].

DOI: 10.17802/2306-1278-2019-8-1-90-99

3. Остроумова О.Д., Извеков А.А., Воеводина Н.Ю. Курение как фактор риска сердечно-сосудистых и цереброваскулярных заболеваний: распространенность, влияние на прогноз, возможные стратегии прекращения курения и их эффективность. Часть 1. Распространенность курения и влияние на прогноз. Рацион. фармакотерапия в кардиологии. 2017; 13 (6): 871-9 [Ostroumova OD, Izvekov AA, Voevodina NYu. Smoking as a risk factor of cardiovascular and cerebrovascular diseases: prevalence, impact on prognosis, possible smoking cessation strategies and their effectiveness. Part 1. Smoking prevalence and impact on prognosis. Rational Pharmacotherapy in Cardiology. 2017; 13 (6): 871-9 (in Russian)]. DOI: 10.20996/1819-6446-2017-13-6-871-879

4. Zhang YJ, lqbal J, van Klaveren D, et al. Smoking is associated with adverse clinical outcomes in patients undergoing revascularization with $\mathrm{PCI}$ or CABG: the SYNTAX trial at 5-year follow-up. J Am Coll Cardiol 2015; 65 (11): 1107-15. DOI: 10.1016/j.jacc.2015.01.014

5. Ruppert AM, Amrioui F, Fallet V, Cadranel J. Peri-operative management of smoking. Rev Pneumol Clin 2018; 74 (3): 154-9. DOI: 10.1016/j.pneumo.2018.04.007

6. Бокерия Л.А., Аронов Д.М. Российские клинические рекомендации. Коронарное шунтирование больных ишемиче- ской болезнью сердца: реабилитация и вторичная профилактика. CardioСоматика. 2016; 7 (3-4): 5-71 [Bokeriya LA, Aronov DM. Russian clinical guidelines Coronary artery bypass grafting in patients with ischemic heart disease: rehabilitation and secondary prevention. Cardiosomatics. 2016; 7 (3-4): 5-71 (in Russian)].

7. Кардиоваскулярная профилактика. 2017. Российские национальные рекомендации. Рос. кардиол. журн. 2018; 23 (6): 7-122 [Cardiovascular prevention. 2017. National guidelines. Russian Journal of Cardiology. 2018; 23 (6): 7-122 (in Russian)]. DOI: 10.15829/1560-4071-2018-6-7-122

8. Pierre S, Rivera C, Le Maître B, et al. Guidelines on smoking management during the perioperative period. Anaesth Crit Care Pain Med 2017; 36 (3): 195-200.

DOI: $10.1016 / \mathrm{j}$. accpm.2017.02.002

9. Musallam KM, Rosendaal FR, Zaatari G, et al. Smoking and the risk of mortality and vascular and respiratory events in patients undergoing major surgery. JAMA Surg 2013; 148 (8): 755-62. DOI: 10.1001/jamasurg.2013.2360

10. Turan A, Mascha EJ, Roberman D, et al. Smoking and perioperative outcomes. Anesthesiology 2011; 114 (4): 837-46. DOI: 10.1097/ALN.0b013e318210f560

11. Grønkjær M, Eliasen M, Skov-Ettrup LS, et al. Preoperative smoking status and postoperative complications: a systematic review and meta-analysis. Ann Surg 2014; 259 (1): 52-71. DOI: 10.1097/SLA.0b013e3182911913

12. Тинюков А.Н., Кудряшов Д.А., Никитин Л.Н. Влияние табакокурения на развитие послеоперационных осложнений. Междунар. студенческий научный вестник. 2017; (2): 25. Режим доступа: http://www.eduherald.ru/ru/article/ view?id=16827. Ссылка активна на 10.06.2020 [Tinyukov AN, Kudryashov DA, Nikitin LN. The effect of smoking on the development of postoperation complications. Mezhdunar. studencheskij nauchnyj vestnik. 2017; (2): 25. Available at: http://www.eduherald.ru/ru/article/view?id=16827. Accessed: 10.06.2020 (in Russian)].

13. Wong J, Abrishami A, Riazi S, et al. A perioperative smoking cessation intervention with varenicline, counseling, and fax referral to a telephone quitline versus a brief intervention: a randomized controlled trial. Anesth Analg 2017; 125 (2): 571-9. DOI: 10.1213/ANE.0000000000001894 
14. Khullar D, Maa J. The impact of smoking on surgical outcomes. J Am Coll Surg 2012; 215 (3): 418-26.

DOI: 10.1016/j.jamcollsurg.2012.05.023

15. Заболотских И.Б., Трембач Н.В., Грицан А.И., и др. Периоперационное ведение пациентов с дыхательной недостаточностью. Анестезиология и реаниматология. 2018; 1-2: 102-16 [Zabolotskikh IB, Trembach NV, Gritsan Al, et al. Perioperative management of patients with respiratory failure. Russian Journal of Anaesthesiology and Reanimatology. 2018; 1-2: 102-16 (in Russian)]. DOI: 10.17116/anaesthesiology201801-021102

16. lida H. Preoperative assessment of smoking patient. Masui 2010; 59 (7): 838-43.

17. Oztürk O, Yılmazer I, Akkaya A. The attitudes of surgeons concerning preoperative smoking cessation: a questionnaire study. Hippokratia 2012; 16 (2): 124-9. Available at: https://www.ncbi.nlm.nih.gov/ pmc/articles/PMC3738413/. Accessed: 10.06.2020.

18. Синдром зависимости от табака, синдром отмены табака у взрослых. Клинические рекомендации. 2018. Режим доступа: http://objects.narcologos.ru/01/000897.pdf. Ссылка активна на 10.06.2020 [Syndrome of dependence on tobacco, tobacco withdrawal syndrome in adults. Clinical recommendations. 2018. Available at: http://objects.narcologos.ru/01/000897.pdf. Accessed: 10.06 .2020 (in Russian)].

19. Duceppe E, Parlow J, MacDonald P, et al. Canadian Cardiovascular Society Guidelines on perioperative cardiac risk asssessment and management for patients who undergo noncardiac surgery. Can J Cardiol 2017; 33 (1): 17-32.

DOI: 10.1016/j.cjca.2016.09.008

20. Nelson G, Altman AD, Nick A, et al. Guidelines for pre- and intraoperative care in gynecologic/oncology surgery: Enhanced Recovery After Surgery (ERASR) Society recommendations Part I. Gynecol Oncol 2016; 140 (2): 313-22.

DOI: 10.1016/j.ygyno.2015.11.015

21. Chaikof EL, Dalman RL, Eskandari MK, et al. The Society for Vascular Surgery practice guidelines on the care of patients with an abdominal aortic aneurysm. J Vasc Surg 2018; 67 (1): 2-77. DOI: 10.1016/j.jvs.2017.10.044

22. Pierre S, Rivera C, Le Maître B, et al. Guidelines on smoking management during the perioperative period. Anaesth Crit Care Pain Med 2017;36 (3): 195-200.DOI: 10.1016/j.accpm.2017.02.002

Статья поступила в редакцию / The article received: 17.02.2021

Статья принята к печати / The article approved for publication: 24.03.2021

Статья опубликована / Article published: 31.03.2021

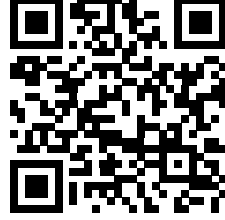

OMNIDOCTOR.RU 